\title{
Cubrimiento radicular con colgajo posicionado coronal y matriz dérmica acelular en recesiones clase II y III de Miller en paciente con diabetes mellitus tipo II. Reporte de caso
}

\author{
Carmen Victoria Sánchez Orozco, ${ }^{*}$ Maritza Espinosa-Arreola, ${ }^{\ddagger}$ Cindy Hernández Romero $§$
}

\section{RESUMEN}

Se considera recesión gingival al desplazamiento del margen gingival hacia apical a la unión cemento-esmalte, exponiendo la superficie radicular al medio oral; es una característica clínica frecuente en la población en general, la cual puede provocar caries radicular, hipersensibilidad dental o una estética dental no favorable. Actualmente son muchas las formas de tratar este tipo de defectos mucogingivales, uno de éstos es el injerto de tejido conectivo, el cual ha tenido excelentes resultados y se considera uno de los mejores; sin embargo, uno de los inconvenientes es realizar en un segundo sitio quirúrgico y la cantidad limitada de injerto. Actualmente se ha introducido el uso de matriz dérmica acelular, con la cual no es necesario un segundo sitio quirúrgico, se reduce el tiempo de trabajo y ofrece mayor confort al paciente. Los beneficios de este material pueden ser extrapolados a pacientes en los cuales la opción de una segunda área quirúrgica no es tan recomendable. El objetivo de este trabajo fue valorar la eficacia clínica del uso de matriz dérmica acelular para el cubrimiento radicular en recesiones clase II y III de Miller utilizando una técnica específica.

Palabras clave: Recesión gingival, cobertura radicular, matriz dérmica acelular, lesiones gingivales no cariosas, colgajo posicionado coronalmente.

\section{INTRODUCCIÓN}

Cada día es más frecuente observar la preocupación de los pacientes por la presencia de recesiones gin-

* Residente de la Especialidad de Periodoncia.

‡ Profesor-Investigador, Centro de Investigación Oncológica Una Nueva Esperanza, Universidad Popular Autónoma del Estado de Puebla (UPAEP).

$\S$ Directora de la Especialidad, Profesora de Clínica de Periodoncia.

Recibido: 07 de septiembre de 2019. Aceptado: 10 de noviembre de 2019.

Este artículo puede ser consultado en versión completa en www.medigraphic.com/periodontologia

\begin{abstract}
Gingival recession is considered to be the displacement of the apical gingival margin to the cement-enamel junction, exposing the radicular surface to the oral environment; It is a common clinical feature in the general population, which can cause root caries, dental hypersensitivity or unfavorable dental aesthetics. Currently there are many ways to treat this type of mucogingival defects, one of these is the connective tissue graft (CTG), which has had excellent results and is considered one of the best, however, one of the drawbacks is to perform a second surgical site and the limited amount of graft. Currently, the use of acellular dermal matrix (ADM) has been introduced, with which a second surgical site is not necessary, the working time is reduced, and it offers greater patient comfort. The benefits of this material can be extrapolated to patients in whom the option of a second surgical area is not recommendable. The objective of this work was to evaluate the clinical efficacy of the use of ADM for root coverage in Miller class II and III recesses using a specific technique.
\end{abstract}

Keywords: Gingival recession, root coverage, acellular dermal matrix, non-carious gingival lesions, coronally advanced flap.

givales (RG) localizadas o generalizadas. Los principales motivos de consulta suelen ser el compromiso estético, la presencia de hipersensibilidad, caries radicular, afracciones o abrasiones. Algunas de las causas son cepillado traumático, malposición dentaria, restauraciones subgingivales, trauma oclusal, movimientos ortodóncicos, frenillos aberrantes, enfermedad periodontal y fenotipo gingival. ${ }^{1}$

Se considera que el tratamiento adecuado de la $\mathrm{RG}$ debe dividirse en dos etapas: etiológica y correctiva, por lo que antes de poder realizar la corrección de este tipo de defectos, es esencial realizar una evaluación clínica y radiográfica para conocer la causa, así como conseguir un diagnóstico, pronóstico y plan de tratamiento acertado. ${ }^{2-4}$ 


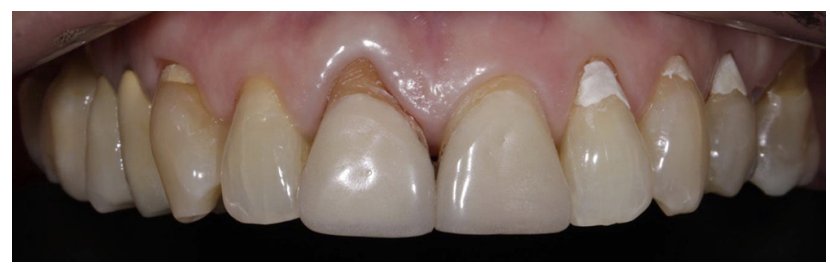

Figura 1: Imagen inicial, órganos dentarios 13, 22, 23 y 24 con ionómeros de vidrio.

El tratamiento quirúrgico para el cubrimiento radicular puede realizarse de tres formas:

1. Colgajos desplazados.

2. Injertos de tejido conectivo (ITC) o injerto gingival libre.

3. Tratamientos adicionales: matriz de proteínas del esmalte o matriz dérmica acelular (MDA).

Con estos últimos, se pueden realizar diferentes técnicas quirúrgicas. Para poder escoger la ideal deben tomarse en cuenta varios aspectos: el número de recesiones, su tamaño, la cantidad y grosor de encía queratinizada y la altura de la papila de los dientes adyacentes..$^{1,5,6}$

El objetivo de este trabajo es determinar el porcentaje de cobertura radicular, así como la evolución de la cicatrización con el uso de MDA en un paciente con diabetes tipo II controlada. ${ }^{7}$

\section{DESCRIPCIÓN DEL CASO CLÍ́NICO}

Mujer de 59 años de edad referida de la clínica de Odontología Restaurativa de la Universidad Popular Autónoma del Estado de Puebla (UPAEP) hacia el Postgrado de Periodoncia de la misma Universidad

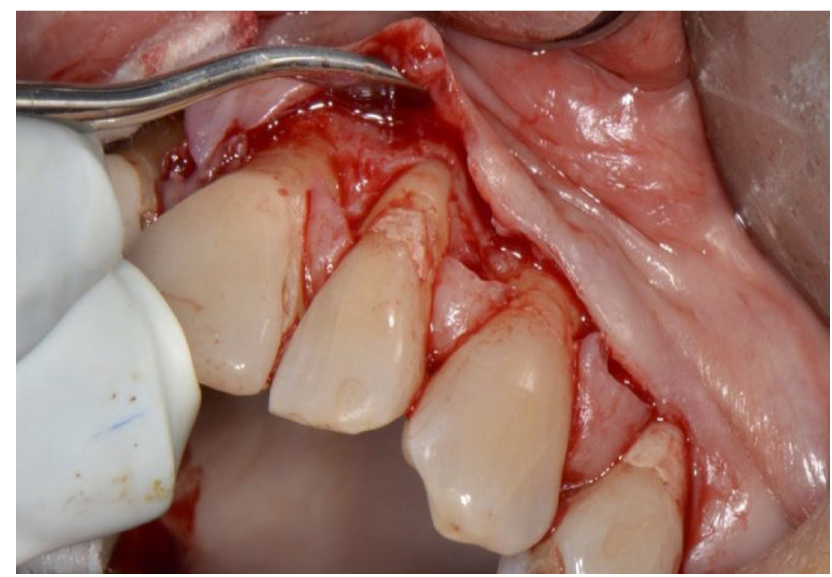

Figura 3: Levantamiento de colgajo a espesor parcial y total.

para cubrimiento radicular de la zona anterosuperior. La paciente refiere como antecedentes personales patológicos diabetes mellitus en control (135 $\mathrm{mg} / 100 \mathrm{~mL})$. A la exploración clínica y radiográfica obtenemos un diagnóstico de gingivitis asociada solamente a biofilm, fenotipo combinado, recesiones clase II y III de Miller, prótesis parcial fija, restauraciones con resina y ligeras giroversiones.

Para evitar la toma de injerto del paladar y brindar mayor confort se ofrece tratamiento con MDA.

El área restaurativa realizó la colocación de ionómero de vidrio en los órganos dentarios que presentaban afracciones con profundidad mayor a $0.5 \mathrm{~mm}$, para tener mejor adhesión de MDA a la superficie radicular (Figura 1). Se toman signos vitales, se realiza asepsia intra y extraoral, bajo infiltración de articaína al 4\% con epinefrina 1:100,000 en fondo de saco y se procedió a realizar el tratamiento quirúrgico.
Figura 2:

Diseño de incisiones oblicuas (A) e intrasulculares (B).
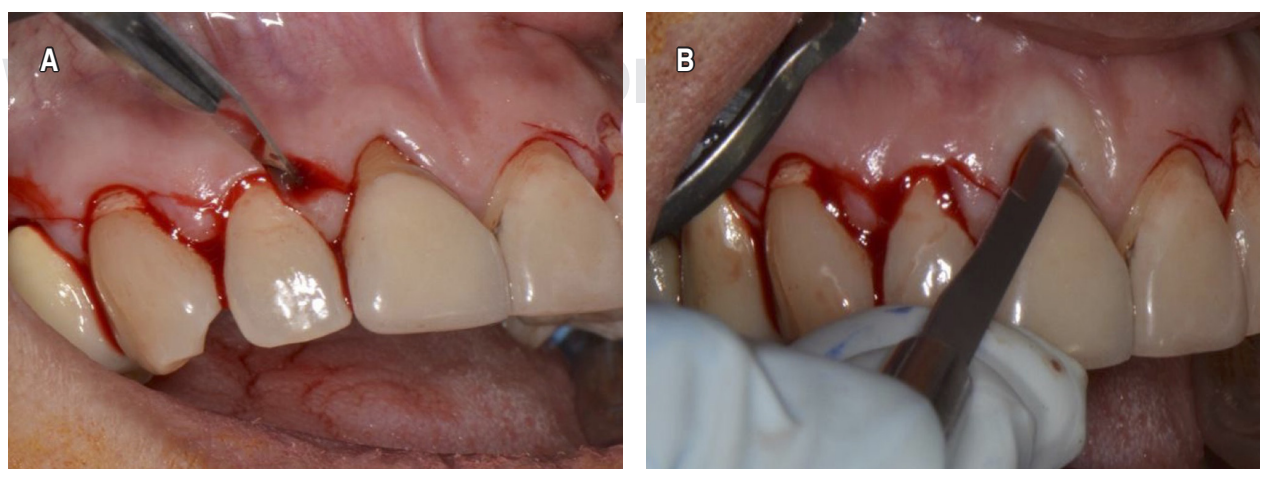
Rev Mex Periodontol 2019; X (3): 49-53

Figura 4:

Unión de dos segmentos para obtener uno solo de $5 \times 40 \mathrm{~mm}$

(A) y sutura de la MDA (B).
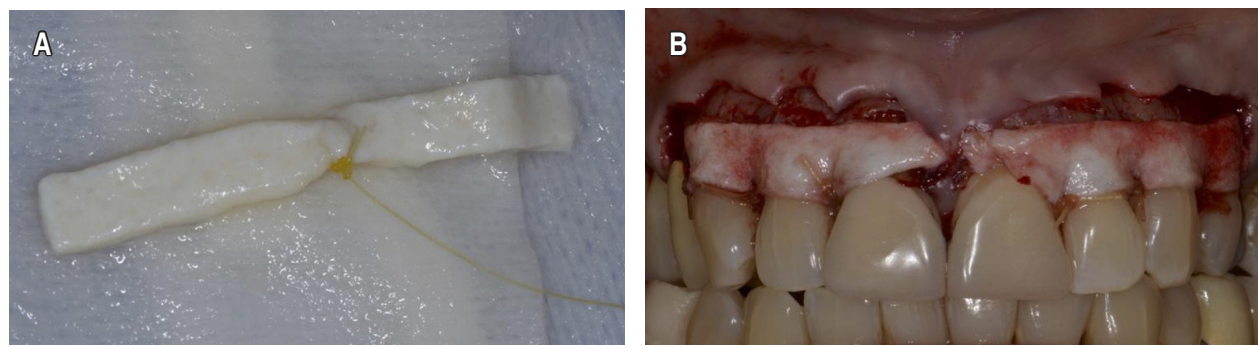

Se empleó la técnica de colgajo posicionado coronal, enfoque frontal (descrita por el Dr. Zuchelli), se realizó una ligera modificación abarcando el área de los caninos y un premolar. Se inició con las incisiones horizontales (oblicuas) comenzando por distal del diente 11, 12, 13, 21, 22, 23 (Figura 2A), después incisiones intrasulculares con microbisturí en diente 11 y 21 proximal mesial, y vestibular de diente 12, 13, 22 y 23 (Figura 2B). Se continuó con la elevación del colgajo a espesor parcial y total para liberarlo de tensión (Figura 3). Se realizó la desepitelización de las papilas, raspado y alisado radicular. Una vez preparado el sitio quirúrgico se continuó con la colocación de la matriz dérmica (OrACELL ${ }^{\circledR}$ LifeNet Health $20 \times 20 \mathrm{~mm}$ ), preparando dos segmentos de $5 \times 20 \mathrm{~mm}$ cada uno y se suturaron para obtener uno solo de $5 \times 40 \mathrm{~mm}$ (Figura 4A), se colocó MDA en el sitio quirúrgico

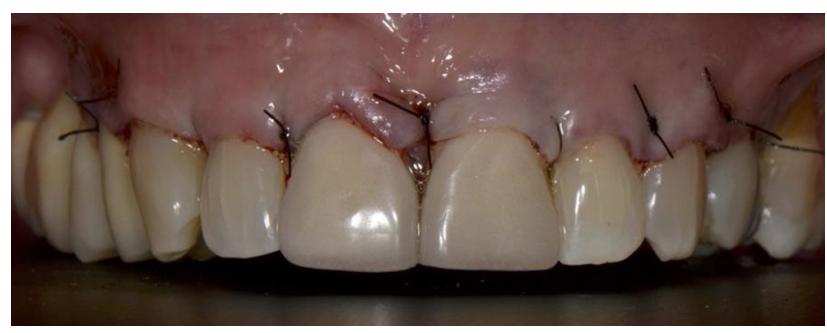

Figura 5: Posicionamiento del colgajo hacia coronal.

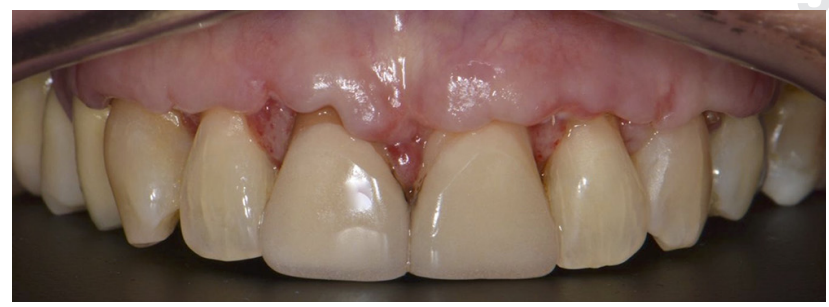

Figura 6: Imagen a los 21 días postoperatorio. y se suturó con cat-gut 4-0 en puntos simples en interproximal (Figura 4B). Por último se posicionó el colgajo coronalmente con nylon 5-0 y puntos en colchonero vertical (Figura 5).

Se le dieron al paciente indicaciones postoperatorias, prescribiendo antibiótico, analgésico, antiinflamatorio y uso de clorhexidina $0.12 \%$ por 15 días. La paciente fue citada a revisión a los ocho, 15 (retiro de puntos) y 21 días postoperatorios (Figura 6). Presentó mínimas molestias, entre ellas dolor e inflamación, la cicatrización no mostró ninguna complicación (infección, necrosis o exposición).

\section{Resultados}

Posterior a los tres meses (Figura 7), a los seis meses (Figura 8) y al año del tratamiento (Figuras

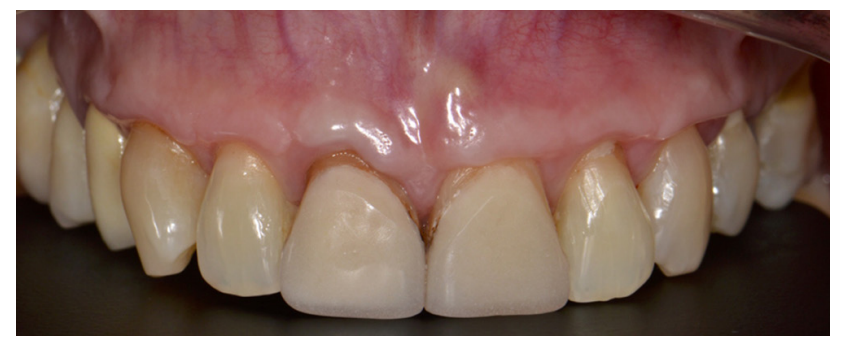

Figura 7: Imagen a los tres meses postoperatorio.

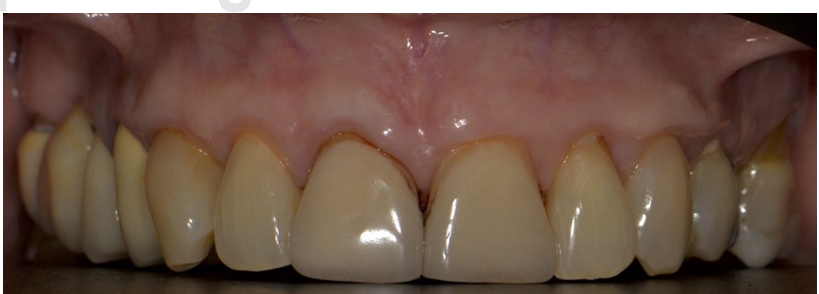

Figura 8: Imagen a los seis meses postoperatorio. 


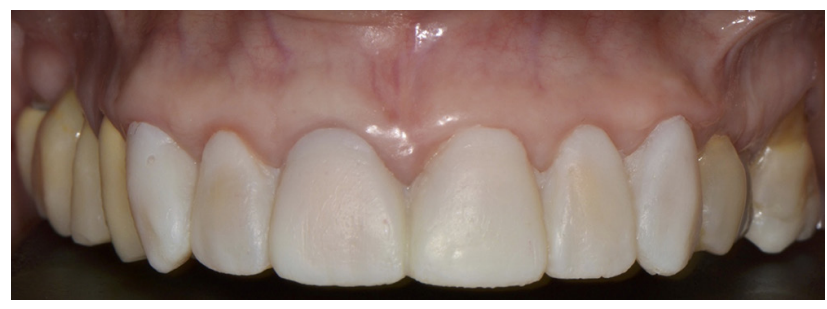

Figura 9: Imagen a los 12 meses postoperatorio con provisionales.

9 y 10), cuatro de las piezas tratadas lograron un recubrimiento radicular al 100\%; sin embargo, dos lograron únicamente $75 \%$. Los resultados de cada órgano dentario se muestran en la Tabla 1. Con la utilización de MDA se obtuvo en promedio un recubrimiento radicular de $2.3 \mathrm{~mm}$ por diente al año del tratamiento.

\section{Discusión}

Los resultados obtenidos fueron similares a los reportados por Marmar Modarressi y Hom-Lay Wang en 2009, donde evaluaron la disminución de la recesión a un año, informando un promedio de 2.79 $\mathrm{mm}$ de cubrimiento en la recesión, cabe señalar que en su estudio se presentó una infección a la semana de la operación; sin embargo, no se reportó afección del injerto. ${ }^{8}$

Aichelmann-Reidy y colaboradores en 2001 demostraron que la capacidad de cubrimiento radicular de MDA no mostró diferencias significativas al compararla con el ITC, el cual se considera el estándar de oro. ${ }^{9}$ En el presente caso no se obtuvo el cubrimiento radicular al 100\% en todos los órganos dentarios debido al tipo de recesión (clase III Miller).

De acuerdo con los estudios realizados por Wei y su equipo, el injerto de MDA produce una menor can-

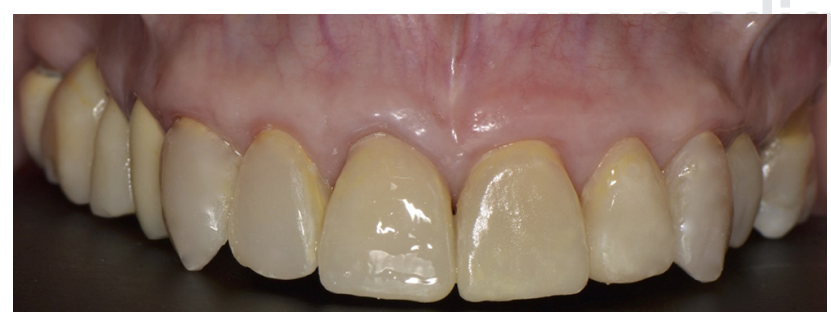

Figura 10: Imagen a los 12 meses postoperatorio con colocación de carillas.
Tabla 1: Resultados por órgano dentario.

\begin{tabular}{ccc} 
OD & $\begin{array}{c}\text { Medida de la RG } \\
\text { prequirúrgica }(\mathrm{mm})\end{array}$ & $\begin{array}{c}\text { Medida de la } \mathrm{RG} \text { un } \\
\text { año después }(\mathrm{mm})\end{array}$ \\
\hline 13 & -2 & 0 \\
11 & -4 & -1 \\
21 & -1 & 0 \\
22 & -3 & 0 \\
23 & -2 & 0 \\
24 & -4 & -1 \\
\hline
\end{tabular}

tidad de encía insertada. ${ }^{10}$ Este caso se ha evaluado únicamente un año después de colocar MDA, por lo que es importante poder tener una revisión a largo plazo para tener una comparativa más sustentada, como lo realizó Barker y su grupo, en donde encontraron una reducción significativa de aproximadamente $80 \%$ de cobertura de raíz después de una evaluación de seis meses para defectos de recesión Miller clase II y III, en donde sus resultados confirman la estabilidad de los injertos de seis a 12 meses. ${ }^{11}$

A pesar de lo reportado en el presente caso clínico, es importante tener en cuenta que los resultados no pueden generalizarse a todos los pacientes con esta misma afección.

\section{Conclusiones}

El uso de MDA parece tener resultados prometedores similares a los encontrados con el uso de ITC, sin embargo, es necesaria la realización de estudios a largo plazo donde se valore la estabilidad de los tejidos utilizando MDA.

La valoración de la recesión, el fenotipo periodontal, la técnica a utilizar y el estado de salud sistémico del paciente, siempre será importante para tener éxito en el tratamiento.

\section{Referencias}

1. Cairo F. Periodontal plastic surgery of gingival recessions at single and multiple teeth. Periodontology 2000. 2017; 75 (1): 296-316.

2. Vicario M, Pascual A, Vives M, Santos A. Técnicas de cirugía mucogingival para el recubrimiento radicular. RCOE. 2006; 11 (1): $61-73$ 
3. Cummings LC, Kaldahl WB, Allen EP. Histologic evaluation of autogenous connective tissue and acellular dermal matrix grafts in humans. J Periodontol. 2005; 76 (2): 178-186.

4. Harris RJ. Root coverage with connective tissue grafts: an evaluation of short- and long-term results. J Pediodontol. 2002; 73 (9): 1054-1059.

5. Mahn DH. Use of the tunnel technique and an acellular dermal matrix in the treatment of multiple adjacent teeth with gingival recession in the esthetic zone. Int J Periodontics Restorative Dent. 2010; 30 (6): 593-599.

6. Zucchelli G. Mucogingival esthetic surgery. Italia: By Quintessenza Edizioni; 2013.

7. Navarro-Sánchez AB, Faria-Almeida R, Bascones-Martínez A. Relación entre diabetes mellitus y enfermedad periodontal. Av Periodon Implantol. 2002; 14 (1): 9-19.

8. Modarressi M, Wang HL. Tunneling procedure for root coverage using acellular dermal matrix: a case series. Int J Periodontics Restorative Dent. 2009; 29 (4): 395-403.

9. Aichelmann-Reidy ME, Yukna RA, Evans GH, Mars HF, Mayer ET. Clinical evaluation of an acelluar allograft dermis for the treatment of human gingival recession. J Periodontol. 2001;72 (8): 998-1005.

10. Wei PC, Laurell L, Geivelis M, Lingen MW, Maddalozzo D. Acellular dermal matrix allografts to achieve increased attached gingiva. Part 1. A clinical study. J Periodontol. 2000; 71 (8): 1297-1305.

11. Barker TS, Cueva MA, Rivera-Hidalgo F, Bach MM, Rossmman JA, Kerns DJ et al. A comparative study of root coverage using two different acellular dermal matrix products. J Periodontol. 2010; 81 (11): 1596-1603.

Correspondencia:

Carmen Victoria Sánchez Orozco

Universidad Popular Autónoma

del Estado de Puebla.

Tel: 22-2189-2818

E-mail:dra_230688@hotmail.com 